\title{
Dual inhibition using cabozantinib overcomes HGF/MET signaling mediated resistance to pan-VEGFR inhibition in orthotopic and metastatic neuroblastoma tumors
}

\author{
ESTELLE DAUDIGEOS-DUBUS ${ }^{1}$, LUDIVINE LE DRET ${ }^{1}$, OLIVIA BAWA ${ }^{2}$, PAULE OPOLON ${ }^{2}$, \\ ALBANE VIEVARD $^{3}$, IRÈNE VILLA ${ }^{4}$, JACQUES BOSQ $^{4}$, GILLES VASSAL $^{1}$ and BIRGIT GEOERGER ${ }^{1}$ \\ ${ }^{1}$ Vectorology and Anticancer Therapies, UMR 8203, CNRS, Univ. Paris-Sud, Gustave Roussy, \\ Université Paris-Saclay, Villejuif; ${ }^{2}$ Preclinical Evaluation Platform, Gustave Roussy, Villejuif; \\ ${ }^{3}$ Tribvn, Châtillon; ${ }^{4}$ Pathology Laboratory, Gustave Roussy, Villejuif, France
}

Received August 8, 2016; Accepted October 6, 2016

DOI: 10.3892/ijo.2016.3792

\begin{abstract}
MET is expressed on neuroblastoma cells and may trigger tumor growth, neoangiogenesis and metastasis. MET upregulation further represents an escape mechanism to various anticancer treatments including VEGF signaling inhibitors. We developed in vitro a resistance model to panVEGFR inhibition and explored the simultaneous inhibition of VEGFR and MET in neuroblastoma models in vitro and in vivo using cabozantinib, an inhibitor of the tyrosine kinases including VEGFR2, MET, AXL and RET. Resistance in IGR-N91-Luc neuroblastoma cells under continuous in vitro exposure pressure to VEGFR1-3 inhibition using axitinib was associated with HGF and p-ERK overexpression. Cabozantinib exhibited anti-proliferative effects in neuroblastoma cells and reduced cell migration in vitro as measured by phase-contrast with IncuCyte system. In vivo, an enhanced number of animals with IGR-N91-Luc metastases was noted following axitinib treatment as compared to control animals. Orally administered cabozantinib per gavage at 30 and $60 \mathrm{mg} / \mathrm{kg} /$ day significantly inhibited tumor growth of orthotopic adrenal IGR-N91-Luc and metastatic IMR-32-Luc xenografts. Antitumor activity was associated with decreased vascularization, inhibition of p-SRC and induction of apoptotic cell death. Activation of the HGF-mediated MET pathway is involved in escape to selective VEGFR inhibition in neuroblastoma suggesting combined inhibition of MET and VEGFR signaling to reduce secondary resistance and enhanced invasiveness.
\end{abstract}

Correspondence to: Dr Birgit Geoerger, Vectorology and Anticancer Therapies, Department of Pediatric and Adolescent Oncology, UMR 8203, CNRS, Univ. Paris-Sud, Gustave Roussy, Université Paris-Saclay, 114 Rue Edouard Vaillant, 94805 Villejuif, France

E-mail: birgit.geoerger@gustaveroussy.fr

Key words: angiogenesis resistance, pediatric tumors, neuroblastoma, hepatocellular growth factor, MET, cabozantinib

\section{Introduction}

The tyrosine kinase receptor c-MET, also called MET or hepatocyte growth factor receptor (HGFR), is the only known receptor for hepatocyte growth factor (HGF) (1). Aberrant MET signaling plays a pivotal role in angiogenesis as well as in tumor cell proliferation, survival and migration (2-4). Several studies suggest that HGF/c-MET signaling promotes angiogenesis directly by stimulating endothelial cells in response to VEGF in various tumor types (5-8). Moreover, MET has been described as an oncogene in different pathologies such as liver cancer (9) and MET gene amplification is reported in 2-3\% of various types of cancer (10).

In patients with neuroblastoma, a pediatric tumor of the neural crest, high concentrations of HGF and elevated soluble VEGF-A were found correlated with higher stage and poor outcome (11). MET is expressed in most neuroblastoma cell lines and HGF stimulated invasion of neuroblastoma cells in vitro and in vivo, and promoted the development of angiogenic neuroblastoma tumors in vivo $(12,13)$. Recent studies in adult cancers suggest that tumor hypoxia induced by anti-angiogenic therapy such as sorafenib leads to increased c-MET activation, cell migration and aggressiveness $(14,15)$ suggesting a combined inhibiting approach to overcome this escape mechanism. Several preclinical studies have explored the use of combined HGF/MET and VEGF/VEGFR signaling inhibition in adult pathologies such as hepatocellular cancer (16-18) or glioblastoma (15) with reduced tumor aggressiveness and metastasis, encouraging the evaluation in neuroblastoma. Cabozantinib (XL-184) is a multi-targeted tyrosine kinase inhibitor which potently inhibits VEGFR and MET signaling (19). The principal targets of cabozantinib are MET, VEGFR2, AXL and RET, but the compound is also reported to inhibit other kinases including KIT, FLT-3 and TEK (19). Cabozantinib has shown effective inhibition of cell proliferation and migration/invasion in vitro (18,20-22). Cabozantinib exhibited inhibition of tumor growth which is mediated by inhibition of angiogenesis and potent anti-metastatic effects in different types of cancer $(18,20,23,24)$. Clinical phase I to III trials have shown antitumor activity in advanced solid tumors 
such as prostate, lung, renal and thyroid cancer, and the agent is approved for the treatment of progressive, metastatic medullary thyroid cancer (25-32).

This study defined HGF/MET signaling activation as an escape mechanism to selective VEGFR inhibition in neuroblastoma and explored the antitumor activity of concomitant inhibition of VEGFR2 and MET in comparison to specific pan-VEGFR inhibition in preclinical neuroblastoma models.

\section{Materials and methods}

Drugs. Cabozantinib (XL-184; kindly provided by Dana Aftab (Exelixis, Inc., San Francisco, CA, USA) was stored as a solid powder. Axitinib (AG-013736, ref A-1107) was purchased from LC Laboratories (Woburn, MA, USA). For in vitro experiments, cabozantinib and axitinib were dissolved in 100\% dimethyl sulfoxide (DMSO) and diluted in complete cell culture. For in vivo experiments, cabozantinib was dissolved daily in water $/ \mathrm{HCl}$ to a final concentration of 3 and $6 \mathrm{mg} / \mathrm{ml}$; axitinib was suspended in 5\% carboxyl methylcellulose to a final concentration of $6 \mathrm{mg} / \mathrm{ml}$.

Cell lines. Luciferase gene transfected IGR-N91 and IMR-32 cells were derived from metastatic neuroblastoma as previously reported (33). IMR-32-Luc and IGR-N91-Luc were maintained in Roswell Park Memorial Institute (RPMI)-1640 medium and Dulbecco's modified Eagle's medium (DMEM), respectively, containing $10 \%$ fetal calf serum (FCS; all from Life Technologies) at $37^{\circ} \mathrm{C}$ and $5 \% \mathrm{CO}_{2}$. Cell lines were regularly tested and found to be free of mycoplasma.

The IncuCyte proliferation and migration phase-contrast imaging assay. For cell proliferation assay, 15,000 IMR-32-Luc and IGR-N91-Luc cells/well were seeded in 96-well plates and incubated for $72 \mathrm{~h}$ with cabozantinib or axitinib at concentrations of $0-10 \mu \mathrm{mol} / 1$ or $0.5 \%$ DMSO. Cell confluence was imaged by phase contrast using the IncuCyte HD system (IncuCyte ${ }^{\mathrm{TM}}$ live-cell). Frames were captured at 4-h intervals from 2 separate regions/well using a x10 objective. Cultures were run three times in at least quadruplicates. Proliferation growth curves were constructed by imaging plates using IncuCyte ${ }^{\mathrm{TM}}$ Zoom software, where growth curves were built from confluence measurements acquired during round-theclock kinetic imaging. Cell migration was evaluated by scratch assays. A scratch was made on confluent monolayers using a 96-pin WoundMaker ${ }^{\mathrm{TM}}$ and incubated with cabozantinib at $5 \mu \mathrm{mol} / \mathrm{l}$ for $48 \mathrm{~h}$. Wound images were automatically acquired and registered by IncuCyte Zoom software system. Data were processed and analyzed using IncuCyte Zoom 96-Well Cell Invasion Software Application Module (all from Essen BioScience, Inc., Ann Arbor, MI, USA). Data are presented as the wound width closure. The rate of wound closure was compared between treated and non-treated conditions.

Western blot analysis. Total tumor lysates were separated electrophoretically and proteins were detected using monoclonal mouse antibody anti-human $\beta$-actin (S125; diluted 1:1,000; Cell Signaling Technology, Danvers, MA, USA), mouse polyclonal anti-human PARP-1 (Ab-2, 1:600; Calbiochem, San Diego, CA, USA), rabbit polyclonal anti-human p-ERK1/2
(Thr202/Tyr204), ERK1/2, p-AKT (Ser473), AKT, p-VEGFR2 (Tyr1175) (19A10), VEGFR2, p-MET (Tyr1234/1235), p-MET (Tyr1249), MET (1:1,000, all from Cell Signaling Technology) as previously described (34).

Angiogenesis and MAPK proteome array. Protein lysates of parental IGR-N91-Luc and axitinib-resistant AxiR cells were subjected to the human angiogenesis and phospho-MAPK array kit (R\&D Systems, Inc., Minneapolis, MN, USA) according to the manufacturer's recommendations.

Experimental in vivo design. Animal experiments were carried out under conditions established by the European Community (Directive 2010/63/UE) and approved by the Comité d'Ethique en Expérimentation Animale n 26 (CEEA26) and the French Ministry (MENESR) (approval number: 00328.01). Antitumor activity was evaluated against orthotopic and systemic neuroblastoma in female Swiss athymic mice, NSG or BalbC RAG $\gamma \mathrm{c}$ mice, established by injection of $10^{6}$ cells into the left adrenal or the tail vein, respectively, under isoflurane anesthesia as previously reported $(33,34)$ and all efforts were made to minimize suffering. Briefly, treatment started when positive bioluminescent signals were detected. Cabozantinib was administered by oral gavage at 30 or $60 \mathrm{mg} / \mathrm{kg} /$ day for a minimum of 14 days and axitinib at $30 \mathrm{mg} / \mathrm{kg} / \mathrm{twice}$ daily (BID) during 29 days; control animals received vehicle. Animals were followed daily for clinical status, weekly for body weight. Antitumor activity was determined using ultrasound and bioluminescence, respectively. Statistical difference was determined using the two-tailed non-parametric Mann-Whitney or Kruskal-Wallis test and Prism ${ }^{\circledR}$ software version 6.00. For pharmacodynamic analysis, primary tumors were harvested $2 \mathrm{~h}$ after the end of treatment at day 29.

Histology and immunohistochemistry. Hematoxylin-eosinsafranin staining was performed on paraformaldehyde fixed tumors for morphology; immunohistochemistry for CD34 and caspase-3 expression was determined using rat anti-mouse CD34 antibody (1:20; Hycult Technologies) and rat anti-human cleaved caspase-3 antibody (1:100; Cell Signaling Technology) as previously reported (34).

\section{Results}

Acquired resistance to selective VEGFRI-3 inhibition in the IGR-N91-Luc-AxiR cell line is associated with HGF overexpression. In order to explore resistance mechanisms to selective VEGFR inhibition we first developed in vitro a resistant cell line, IGR-N91-Luc-AxiR, through chronic exposure of IGR-N91-Luc cells in culture to the VEGFR1-3 tyrosine kinase inhibitor axitinib. Axitinib at the 50\% growth inhibiting concentration $\left(\mathrm{IC}_{50}\right.$ of $\left.0.75 \mu \mathrm{mol} / \mathrm{l}\right)$ allowed maintaining cell viability as well as selection of resistant clones in the cell line. Constant resistant behavior was achieved after five consecutive passages under continuous axitinib exposure. In regard to cell proliferation inhibition, the $\mathrm{IC}_{50}$ of axitinib in the parental IGR-N91-Luc cells was $0.75 \mu \mathrm{mol} / 1$ when administered once as measured by phase-contrast imaging during $72 \mathrm{~h}$. In contrast, proliferation of the resistant IGR-N91-LucAxiR cells was less affected with an $\mathrm{IC}_{50}$ of $3 \mu \mathrm{M}$ (Fig. 1A). 

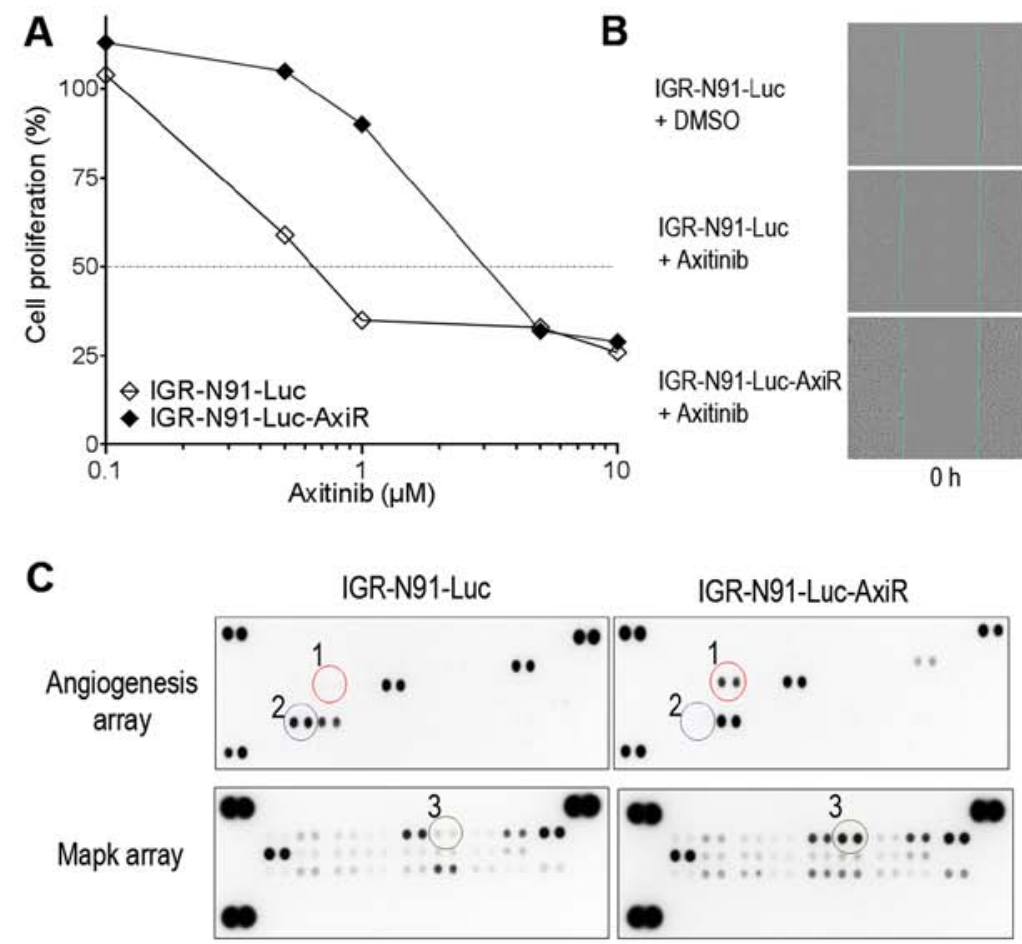

B

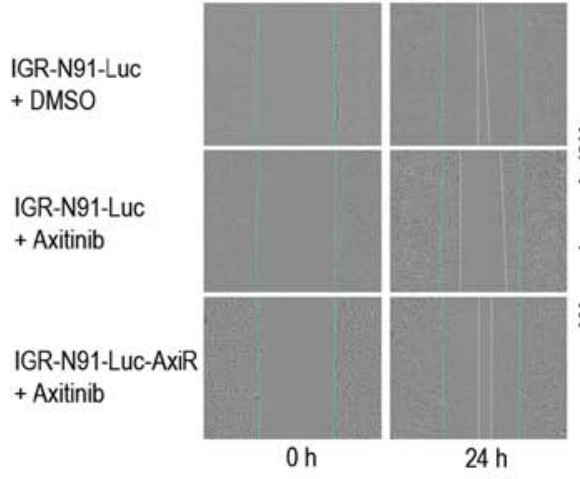

D

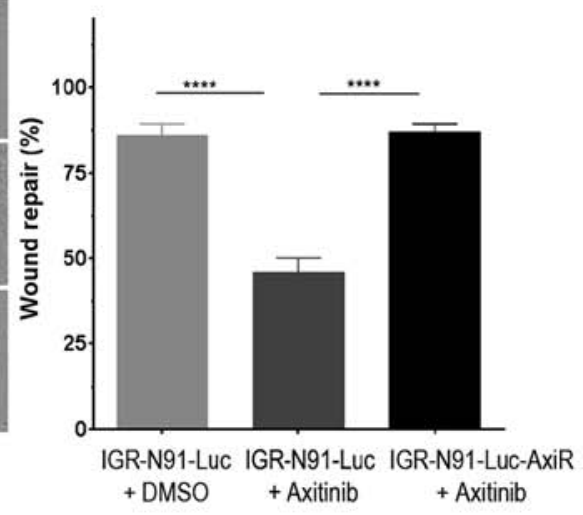
IGR-N91-Luc IGR-N91-Luc-AxiR

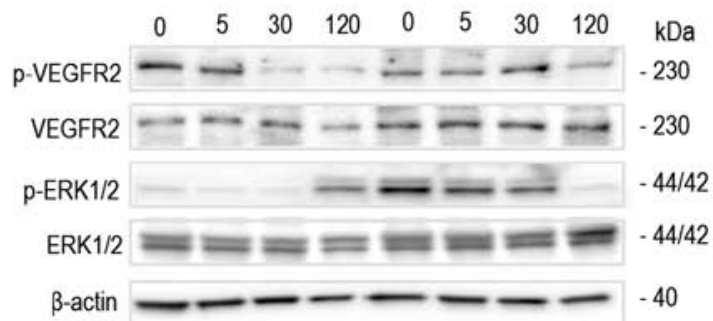

Figure 1. Resistance to axitinib in IGR-N91-Luc-AxiR. IGR-N91-Luc cells were continuously exposed to axitinib at the IC $\mathrm{C}_{50}$ of $0.75 \mu \mathrm{M}$ for 6 weeks. (A) Cell proliferation of parental (open diamonds) and IGR-N91-Luc-AxiR cells (closed diamonds) was measured by phase-contrast imaging under axitinib at indicated concentrations for $72 \mathrm{~h}$. (B) Migration of IGR-N91-Luc and IGR-N91-Luc-AxiR cells treated with axitinib or DMSO was followed by phase-contrast imaging during $24 \mathrm{~h}$. Images represent the wound disclosure of parental IGR-N91-Luc and axitinib-resistant IGR-N91-Luc-AxiR cells under DMSO and axitinib, respectively at baseline and $24 \mathrm{~h}$. Graph shows arithmetic means ( \pm standard error of mean) of percentage of wound repair. Statistical significance was estimated by Kruskal-Wallis test, ${ }^{* * * *} \mathrm{P}<0.0001$. (C) Total lysates of IGR-N91-Luc and IGR-N91-Luc-AxiR cells were subjected to proteome array. Pictures represent angiogenesis array (upper panels) and MAPK array (lower panels). Circles highlight protein expression: 1) HGF; 2) PEDF; and 3) p-ERK-1. (D) Total lysates of IGR-N91-Luc and IGR-N91-LUC-AxiR treated with axitinib during 0, 5, 30 and 120 min were subjected to western blot analyses using antibodies against phosphorylated (p-) and non-phosphorylated VEGFR2 and ERK1/2; $\beta$-actin was used as a reference.

Indeed, growth of IGR-N91-Luc-AxiR cells under axitinib treatment was identical to that of the non-treated parental IGR-N91-Luc cells (data not shown). We further explored the migration capacity of the parental and axitinib-resistant IGR-N91-Luc cells under axitinib treatment. Cells were seeded in monolayers into 96-well plates and a scratch was done when they were at confluence. Axitinib at the $\mathrm{IC}_{50}$ or $0.5 \% \mathrm{DMSO}$ were added into each well and migration was followed during $48 \mathrm{~h}$ when the wound of non-treated parental IGR-N91-Luc control cells was nearly closed. For parental IGR-N91-Luc cells wound repair inhibition of up to $54 \%$ was observed under axitinib treatment whereas IGR-N91-Luc-AxiR cells showed no reduction in cell migration under axitinib and had nearly closed the scratch wound similar to non-treated cells (Fig. 1B). Thus, axitinib-resistant IGR-N91 neuroblastoma cells exhibit reduced sensitivity to inhibition of both growth proliferation and cell migration to axitinib. To explore potential escape mechanisms involved in this resistance we subjected the cell lysates of parental IGR-N91-Luc and IGR-N91-Luc-AxiR cells to proteome arrays for angiogenesis protein and MAPK signaling expression which simultaneously detect the relative levels of 55 angiogenesis-related proteins and the relative phosphorylation of 24 kinases captured by 26 different antibodies, respectively, spotted in duplicates on a nitrocellulose membrane (Fig. 1C). IGR-N91-Luc-AxiR cells exhibited overexpression of HGF and phosphorylation of downstream effector ERK1 as compared to the parental cells as well as reduced expression of PEDF, a physiological negative regulator of angiogenesis. In response to axitinib treatment at $0.75 \mu \mathrm{mol} / 1$, the parental IGR-N91-Luc cells showed inhibition of VEGFR2 phosphorylation up to $120 \mathrm{~min}$ with an activation of p-ERK at the latest time-point explored. In contrast, IGR-N91-Luc-AxiR cells exhibited activated/phosphorylated ERK at baseline, consistent with the MAPK array, which diminished at the latest time-point and no reduction of VEGFR2 phosphorylation was observed to axitinib exposure (Fig. 1D). Thus, activation of the HGF/MET and MAPK signaling pathway is involved in resistance to VEGFR inhibition in neuroblastoma.

HGF stimulates the MET signaling pathway in neuroblastoma cells and cabozantinib inhibits proliferation and migration. We next explored the effects of HGF stimulation on the two parental IGR-N91-Luc and IMR-32-Luc neuroblastoma cell lines. Cells cultured under serum-free medium conditions overnight were incubated with $60 \mathrm{ng} / \mathrm{ml} \mathrm{HGF}$ and harvested at indicated time-points up to $120 \mathrm{~min}$ for western blot analyses (Fig. 2A). In the IMR-32-Luc cells HGF exposure resulted in significant activation of its receptor MET with an increase of phosphorylation in sites Tyr1234/1235 and to a lesser extent at Tyr1349 whereas expression levels of the total receptor remained stable. The effects were less prominent in the IGR-N91-Luc cells. Based on the hypothesis 

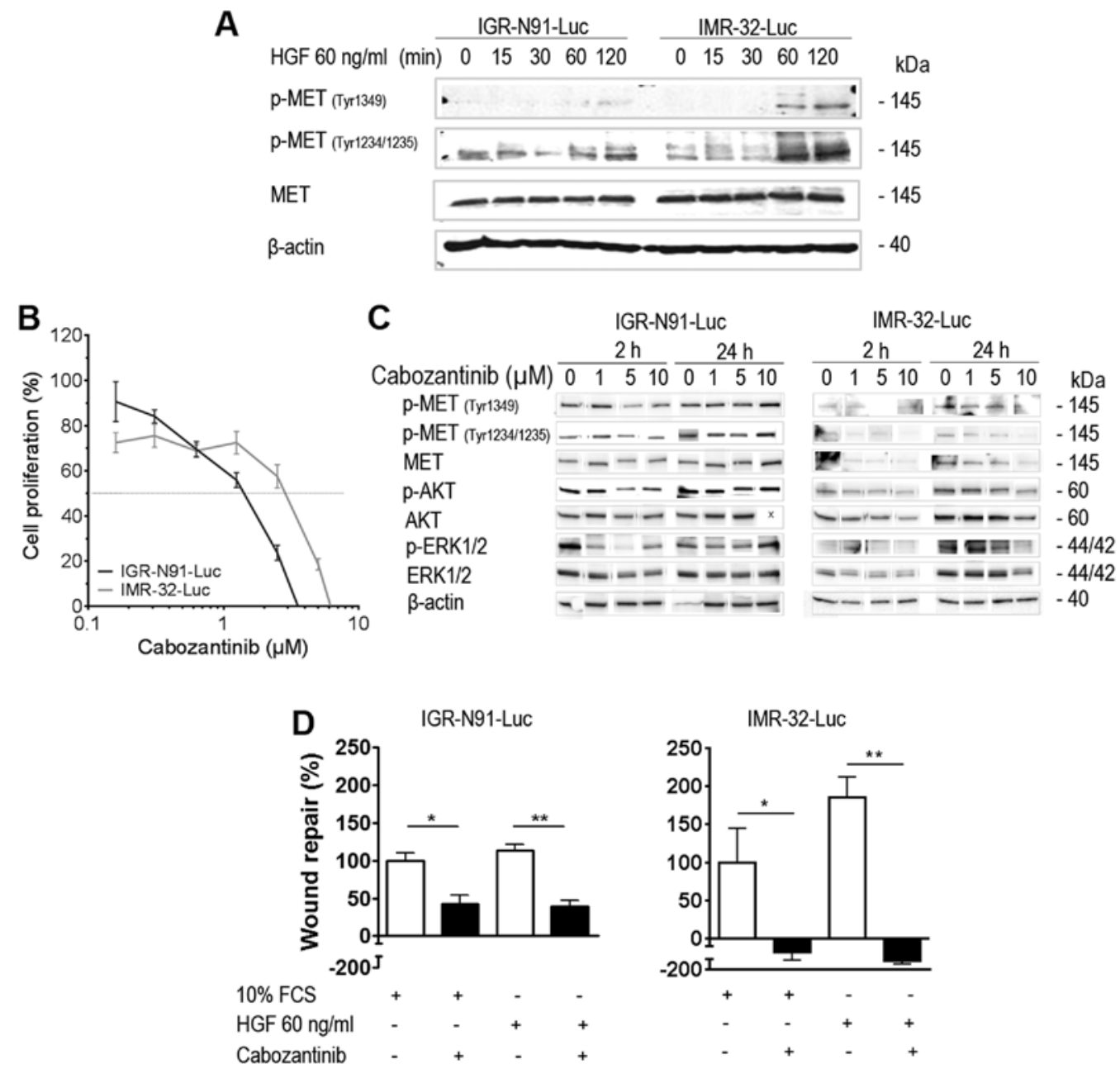

Figure 2. HGF activates MET signaling in neuroblastoma cells which is inhibited by cabozantinib. (A) IGR-N91-Luc and IMR-32-Luc neuroblastoma cell lines were exposed to HGF at $60 \mathrm{ng} / \mathrm{ml}$ for $120 \mathrm{~min}$. Total cell lysates of cells harvested at indicated time-points were subjected to western blot analysis using antibodies against phosphorylated (p-) and non-phosphorylated MET. (B) IGR-N91-Luc and IMR-32-Luc were exposed to cabozantinib at the indicated concentrations and cell proliferation was measured at $72 \mathrm{~h}$ by phase-contrast imaging. (C) IGR-N91-Luc and IMR-32-Luc cells were exposed to cabozantinib at the indicated concentrations and harvested at 2 and $24 \mathrm{~h}$. Total cell lysates were subjected to western blotting using antibodies against phosphorylated (p-) and non-phosphorylated MET, AKT, MAPK and $\beta$-actin. (D) IGR-N91-Luc and IMR-32-Luc were exposed to cabozantinib at $5 \mu \mathrm{M}$ in $10 \%$ FCS or $100 \mathrm{ng} / \mathrm{ml}$ HGF conditions and inhibition of cell migration was measured at $48 \mathrm{~h}$ by phase-contrast imaging. Statistical significance was estimated by Kruskal-Wallis test, ${ }^{*} \mathrm{P}<0.05,{ }^{* *} \mathrm{P}<0.01$.

raised above that HGF signaling is involved in promoting cell survival under selective VEGFR inhibition, we subjected both parental cell lines to the potent VEGFR2 and MET tyrosine kinase inhibitor cabozantinib to the proliferation assay for $72 \mathrm{~h}$. Cabozantinib inhibited cell growth of IGR-N91-Luc and IMR-32-Luc cells in a concentration-dependent manner, with $\mathrm{IC}_{50}$ doses of 1.4 and $2.8 \mu \mathrm{M}$, respectively (Fig. 2B). Despite the similar $\mathrm{IC}_{50}$ of the cells, treatment with cabozantinib at 5 and $10 \mu \mathrm{M}$ for 2 and $24 \mathrm{~h}$ resulted in inhibition of phosphorylation of MET and downstream signaling effectors AKT and MAPK in IMR-32-Luc cells, whereas the inhibition was found only at higher concentrations and at $2 \mathrm{~h}$ in the IGR-N91-Luc cells (Fig. 2C). Effects of selective HGF stimulation on cell proliferation capacity were impossible to evaluate due to the lack of growth of these neuroblastoma cells in the absence of serum. When exploring the effects on migration in both cell lines we found no significant increase in cell migration to HGF selective medium as compared to $10 \%$ FCS growth conditions although IMR-32-Luc cells seemed stimulated under HGF (ns).
Cabozantinib at $5 \mu \mathrm{M}$ reduced significantly migratory capacity in both cell lines, under both selective HGF and serum conditions, and migration was completely inhibited in IMR-32-Luc (Fig. 2D). Thus, activation of HGF-mediated MET signaling is involved in neuroblastoma cell migration and inhibition of MET results in reduced tumor cell migration capacity $(1,8,9)$.

Specific inhibition of VEGFR1-3 signaling pathway is associated with increased metastases of IGR-N91-Luc neuroblastoma tumors. In vivo we first explored pan-VEGFR inhibition using axitinib against the orthotopic IGR-N91-Luc neuroblastoma xenograft model in BalbC RAG $\gamma \mathrm{C}$ mice in order to extend our previous data in the subcutaneous and orthotopic IGR-N91 xenografts that had shown growth inhibition to axitinib (35). Axitinib treatment was initiated 2 days after injection of cells into the left adrenal administered by oral gavage twice daily at $30 \mathrm{mg} / \mathrm{kg} /$ dose and was given for a longer time period (i.e. 29 days) than the previous experiments in order to allow potential development of metastases in 

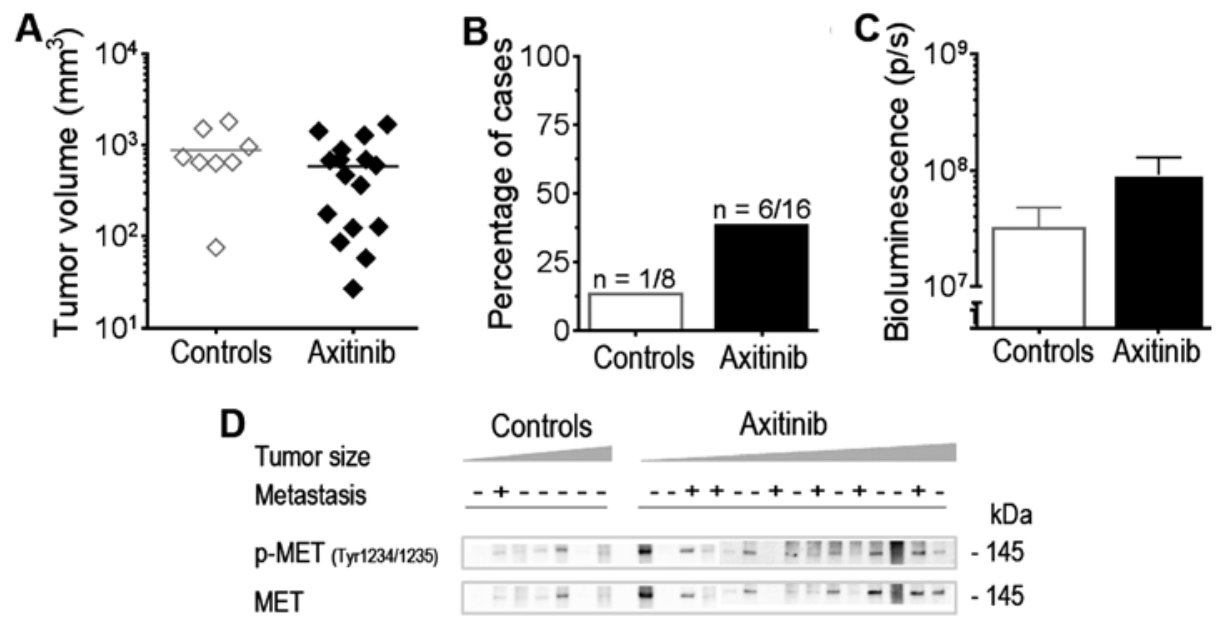

VEGFR1

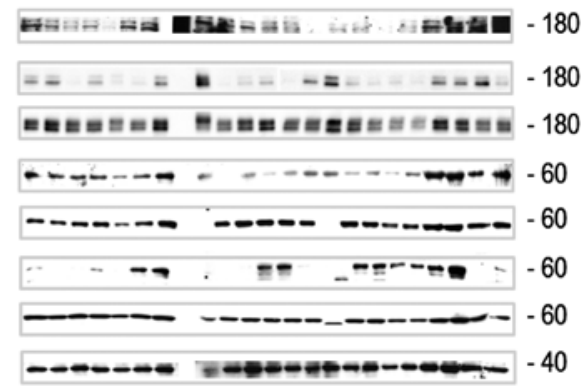

Figure 3. Axitinib against orthotopic IGR-N91-Luc xenograft. Orthotopic IGR-N91-Luc bearing mice were treated orally with axitinib at $30 \mathrm{mg} / \mathrm{kg}$ twice daily ( $\mathrm{n}=16$, closed diamonds) or vehicle ( $\mathrm{n}=8$, open diamonds) for 29 days. (A) Tumor volumes were measured by ultrasound. Graph represents individuals (diamonds) and arithmetic means (horizontal line) of tumor volumes in animals per group. Autopsy was performed at day 29 and presence of metastasis was explored macroscopically and by bioluminescence ex vivo. Graph shows the number of cases which presented metastases (B) and mean \pm standard error of mean of luciferase activity in the liver (C). (D) Primary tumors were subjected to western blot analysis using antibodies against phosphorylated (p-) and nonphosphorylated MET, VEGFR1, ERK1/2, SRC, AKT and $\beta$-actin. Grey triangles represent the increasing primary tumor volumes; (-) represents absence of metastases and (+) presence of metastases in each animal.

this model. At day 29, several animals in the axitinib treated group had reduced tumor load although the median tumor volume of primary adrenal tumors was not significantly reduced compared to controls as determined by ultrasound (Fig. 3A). At autopsy at day 29, macroscopically visible metastases were detected in 6 out of 16 animals treated with axitinib, located in the liver, whereas this was the case in 1 out of 8 control animals (Fig. 3B). Bioluminescence imaging ex vivo detected a signal increase in tumor burden in the livers of treated animals as compared to the treated animals (ns; Fig. 3C). Western blot analysis of adrenal tumors of 7 control and 14 treated animals revealed enhanced MET and ERK phosphorylation in some tumors, independent of tumor size and the occurrence of metastasis. AKT phosphorylation was inhibited in smaller samples in comparison to controls (Fig. 3D). In addition 10 out of the 15 analyzed tumors treated showed SRC phosphorylation which was present only in 2 out of 7 controls. Thus, selective VEGFR1-3 inhibition may result in enhanced metastatic spread and SRC activation in neuroblastoma tumors.

Cabozantinib inhibits tumor growth of orthotopic IGR-N91-Luc neuroblastoma in a dose-dependent manner. Based on our observation in vitro that HGF-mediated MET upregulation could be involved in the invasiveness of neuroblastoma cells, we investigated the dual inhibition of VEGFR and MET against the adrenal IGR-N91-Luc model in NSG mice.
Cabozantinib at 30 and $60 \mathrm{mg} / \mathrm{kg}$ resulted in a dose-dependent tumor growth inhibition of 60 and $87 \%$, respectively, compared to the control group ( $\mathrm{P}=0.005$; Kruskal-Wallis test) with 7 animals in each group (Fig. 4A-C). Protracted administration of cabozantinib at both doses was well tolerated with minor body weight loss in treated animals. To gain insight into the mechanisms by which cabozantinib inhibits tumor growth, we explored anti-angiogenic and pro-apoptotic effects in adrenal tumors using immunohistochemistry and western blot analysis. Cabozantinib treated xenografts showed significantly reduced microvessel density compared to control tumors as determined by $\mathrm{CD} 34$ staining $(\mathrm{P}=0.0116$ for $30 \mathrm{mg} / \mathrm{kg}$ and $\mathrm{P}=0.0025$ for $60 \mathrm{mg} / \mathrm{kg}$ treated tumors; Kruskal-Wallis test; Fig. 4D). A dose-dependent increase in cleaved caspase-3-positive nuclei was noted for cabozantinib at the higher dose as compared to controls ( $\mathrm{P}=0.0091$ for $60 \mathrm{mg} / \mathrm{kg}$; Fig. 4E). When investigating the effects on the VEGF and HGF signaling pathways by exploring known key downstream effectors (Fig. 4F), we observed the appearance of ERK1/2 phosphorylation in tumor samples to cabozantinib treatment as compared to controls. No modification was observed in regard to AKT activation levels. In contrast, SRC phosphorylation was found inhibited at both concentrations in comparison to control tumors.

Thus, cabozantinib acts by inhibition of angiogenesis at both dose levels whereas induction of cell death demands higher concentrations; inhibition of SRC appears associated with antitumor activity. 

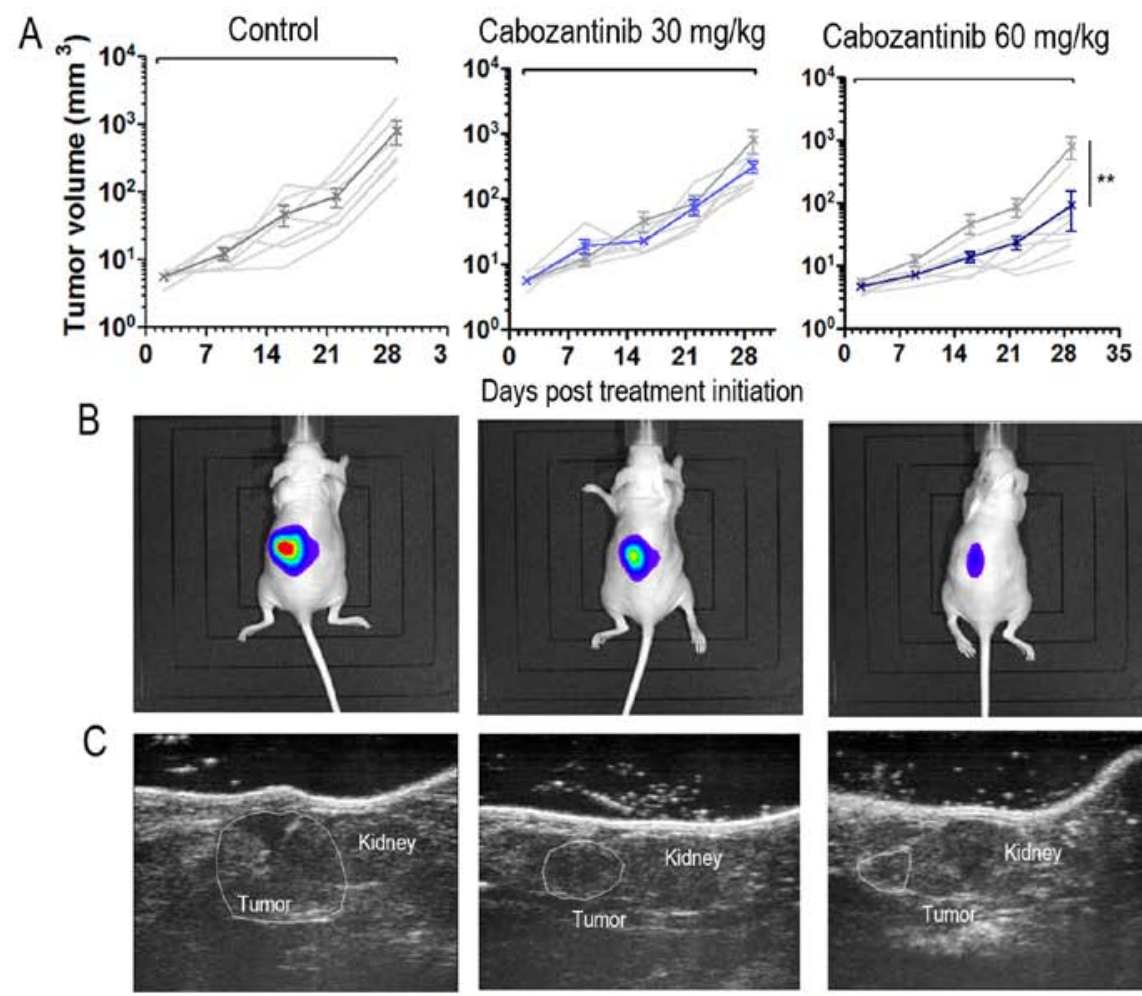

Days post treatment initiation
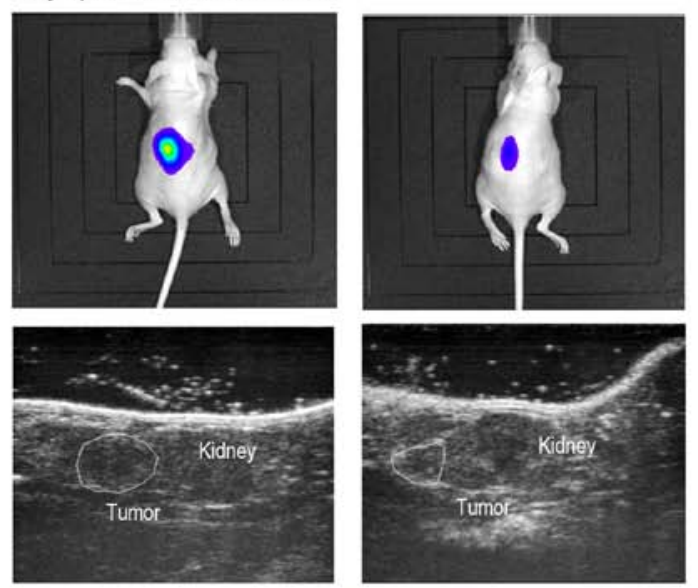

D
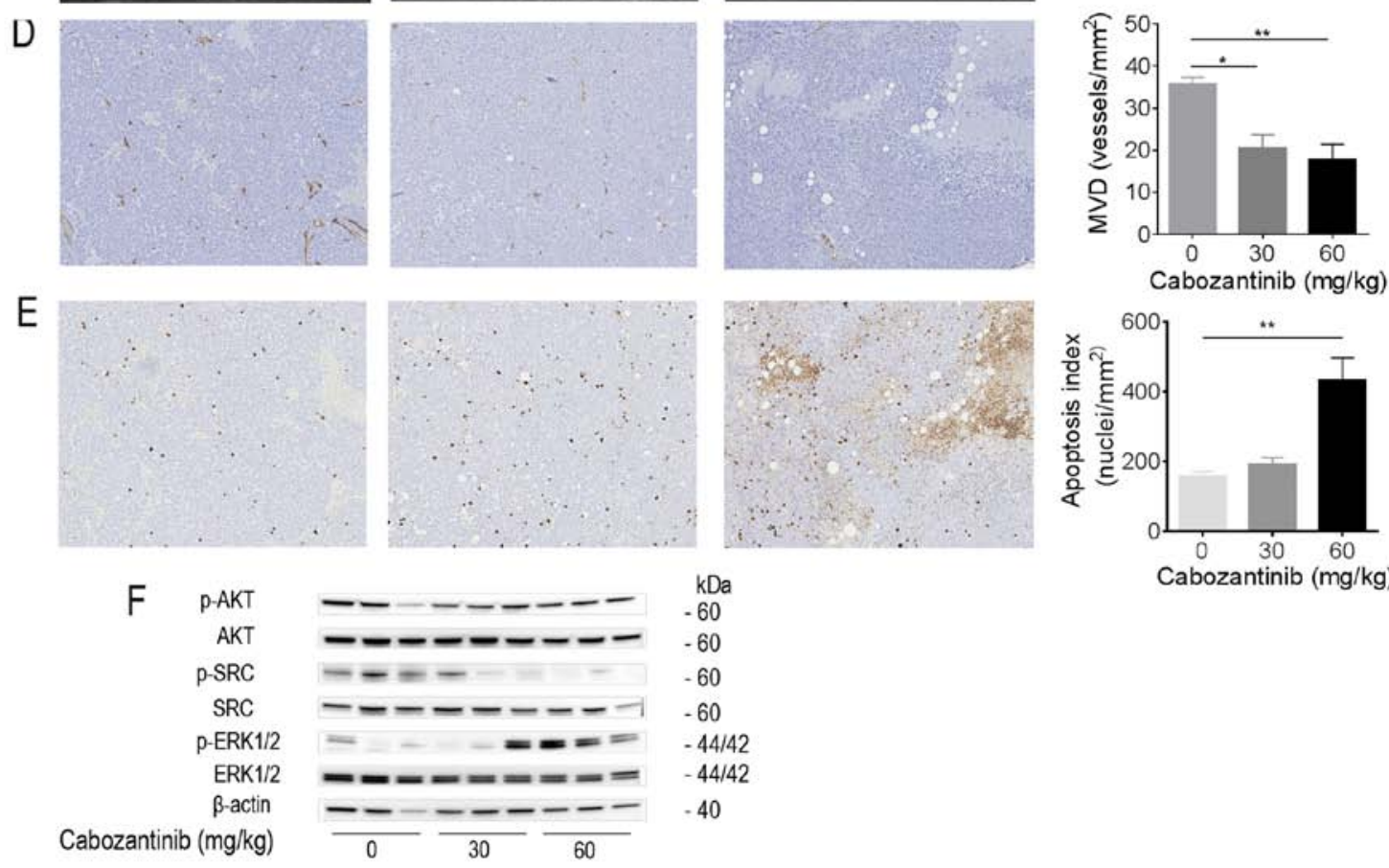

Cabozantinib (mg/kg)

Figure 4. Cabozantinib exhibits antitumor activity against IGR-N91-Luc orthotopic neuroblastoma mediated by anti-angiogenic effects and induction of cell death. Animals bearing orthotopic adrenal IGR-N91-Luc tumors were treated orally with cabozantinib at $30 \mathrm{mg} / \mathrm{kg} / \mathrm{day}$ (light blue line), $60 \mathrm{mg} / \mathrm{kg} / \mathrm{day}$ (dark blue line) or vehicle (grey line). (A) Graphs show growth curves of individual mice (light grey) and arithmetic means ( \pm standard error of mean). Tumor volumes were detected by bioluminescence (B) and ultrasonography (C). Images depict representative adrenal tumors of control and treated animals at day 22. Paraffin-embedded sections of IGR-N91-Luc orthotopic primary tumors treated with vehicle (light grey, $\mathrm{n}=7$ ), cabozantinib at $30 \mathrm{mg} / \mathrm{kg}$ (dark grey, $\mathrm{n}=7$ ) or $60 \mathrm{mg} / \mathrm{kg}$ (black, $\mathrm{n}=6$ ), harvested at day 29 post treatment initiation, were stained immunohistochemically with anti-CD34 and anti-cleaved caspase-3 antibodies. (D) The percentage of CD34-positive area was determined and microvessel density was quantified using CaloPix software and statistically evaluated by Kruskal-Wallis test. (E) The apoptosis index was determined as number of cleaved caspase-3 positive cells per mm². Representative examples of histological stainings are shown at x100 magnification. Positive staining appears as brown color. Graphs represent means $( \pm$ standard error of mean, KruskalWallis test; ${ }^{*} \mathrm{P}<0.05,{ }^{* *} \mathrm{P}<0.01$ ). (F) Total lysates from three individual tumors were subjected to western blotting for expression analyses of phosphorylated (p-) and non-phosphorylated AKT, ERK1/2, SRC and $\beta$-actin.

Cabozantinib reduces metastatic spread in the systemic IMR-32-Luc neuroblastoma model. To be able to best explore the inhibition of metastatic spread of neuroblastoma, we used our systemic IMR-32-Luc model in female NSG mice which develop metastases preferably in lungs, liver and bones (33). Cabozantinib orally at 30 and $60 \mathrm{mg} / \mathrm{kg} / \mathrm{day}$ was initiated at 
A
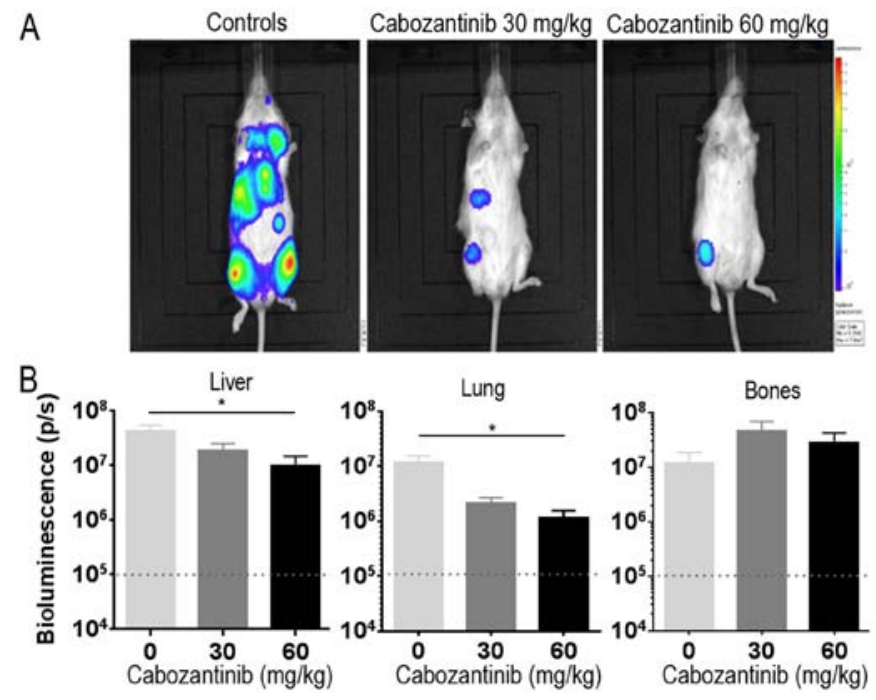

Figure 5. Cabozantinib reduces lung and liver metastases in systemic IMR32-Luc NB. (A) $10^{6}$ IMR-32-Luc cells were injected intravenously in NSG mice into the tail vein. Cabozantinib or vehicle was administered orally once a day at 30 and $60 \mathrm{mg} / \mathrm{kg}$ for 34 days ( $\mathrm{n}=6$ each group). Tumor spread was followed once a week by bioluminescence imaging with IVIS 50 system. (B) Liver, lungs and bones of mice were harvested when the first clinica symptoms appeared and submitted to bioluminescence imaging ex vivo. Graphs represent means \pm standard error of mean. Statistical significance was estimated by Kruskal-Wallis test, ${ }^{*} \mathrm{P}<0.05$.

day 10 post tail vein injection, when bioluminescence signals were detectable in all animals and was given for 34 days when the animals were sacrificed. Bioluminescence signals in vivo in control animals were distributed over the liver region, thorax, and bilateral lower extremities consistent with the previous metastatic homing pattern in this model (33). Cabozantinib treated animals in treatment groups exhibited reduced bioluminescence signals (Fig. 5A). Luciferase activity of tumor cells ex vivo was significantly reduced in liver $(n=6, P=0.0238$; Kruskal-Wallis test) and lung $(n=6$, $\mathrm{P}=0.0141)$ in the $60 \mathrm{mg} / \mathrm{kg}$ treated animals as compared with the control group. This was not the case in bones (Fig. 5B). Thus, cabozantinib reduces metastatic spread to visceral organs in IMR-32 neuroblastoma.

\section{Discussion}

Inhibition of angiogenesis is significantly hampered by the induction of pro-angiogenic factors resulting in secondary tumor escape from treatment. In the present study, we explored the escape mechanisms to selective pan-VEGFR inhibition in preclinical neuroblastoma models. We found HGF mediated MET, MAPK and SRC pathway signaling involved in neuroblastoma escape to VEGFR1-3 inhibition which resulted in enhanced cell migration, suggesting that dual inhibition of VEGFR and MET may be a rational approach for further investigation in neuroblastoma treatment.

Through a new resistant cell line that was developed in vitro by consistent exposure to axitinib we could show that the loss of sensitivity to selective VEGFR1-3 inhibition was associated with the induction of HGF and subsequent signaling through downstream effector MAPK pathway. The HGF/MET pathway is not constitutively upregulated in neuroblastoma but most neuroblastoma cell lines tested exhibited low MET expression (data not shown) which could be stimulated by its physiological ligand HGF, as shown here for the two cell lines IGR-N91-Luc and IMR-32-Luc. MET signaling is involved in cell proliferation as well as cellular migration (36). Hecht et al $(12,13)$ provided the first evidence that the HGF/c-Met pathway is essential for invasiveness and malignant progression of human neuroblastomas in vitro and in chick chorioallantoic membranes. Elevated concentrations of HGF and soluble VEGF-A have been reported in patients with neuroblastoma and were correlated with higher stage disease (11). Both of our luciferase transfected cell lines are sensitive to proliferation and migration inhibiting effects of cabozantinib, an inhibitor of VEGFR2 and MET kinases, with $\mathrm{IC}_{50} \mathrm{~s}$ in the low micromolar range. Our findings were consistent with the recent results described by Zhang et al (37) where IMR-32 was one of the most sensitive neuroblastoma cell lines tested. In addition, we demonstrated that wound repair capacity was significantly reduced in IGR-N91-Luc cells and more importantly in the IMR-32-Luc cell line. This cell line also showed enhanced MET activation/phosphorylation when stimulated with HGF as compared to IGR-N91-Luc suggesting differences in the capacity of neuroblastoma cells to activate this survival pathway. The in vitro effects of cabozantinib were associated with inhibition of MET.

In vivo we confirmed our previous data on tumor growth inhibition with the pan-VEGFR inhibitor axitinib in IGR-N91-Luc neuroblastoma (35). However, in the more immunocompromized BalbC RAG $\gamma \mathrm{C}$ mice, we observed a limited growth inhibiting effect on the primary adrenal tumor whereas animals treated with axitinib presented metastases more frequently at study end, mainly to the liver, suggesting actually the induction of a more aggressive disease pattern under axitinib treatment. When exploring tumor samples, we found, beside the induction of MET, SRC activation/ phosphorylation in most axitinib treated tumors as compared to controls. Both, activation of MET and SRC, have been reported to increase aggressiveness of tumor cells and promote metastases in response to anti-VEGF antibody and multi-tyrosine kinase VEGFR inhibitors such as sorafenib, sunitinib and bevacizumab $(15,24,38,39)$. Moreover, the HGF/ MET signaling has been described to induce angiogenesis independently of VEGF (40). We further detected in our resistant cell line the reduction of expression levels of PEDF, which is a physiological negative regulator of angiogenesis. Loss of PEDF could be another mechanism of resistance in neuroblastoma and its role needs to be explored further.

Consistent with the in vitro evaluation, cabozantinib resulted in significant dose-dependent inhibition of tumor growth of adrenal IGR-N91-Luc tumors compared to controls. These effects appeared more prominent as those observed in the previous experiments to axitinib (35) although the results in sensu stricto cannot be compared directly as they were performed in independent experiments. Orthotopic primary IGR-N91-Luc tumors showed reduced microvessel density for the 30 and the $60 \mathrm{mg} / \mathrm{kg}$ dose levels, however, at the higher treatment dose also significant induction of apoptotic cell death was observed. Prolonged exposure of cabozantinib resulted in a reduction of SRC phosphorylation in treated tumors. SRC activation was detected in tumors of mice following prolonged 
axinitinib treatment and, thus, its inhibition may be additionally involved in the superior effects of cabozantinib albeit it is not inhibiting SRC directly. In our systemic neuroblastoma model, cabozantinib treatment resulted in significant reduction of metastasis formation in the IMR-32-Luc, mainly to the liver and the lungs. However, we observed no reduction of bone metastases as it had been reported in patients with metastatic prostate cancer and the difference of the mouse environment to the human microenvironment could underlie these distinct results in an organ which is very much influenced by the microenvironment $(26,28)$. Recently, it has been shown that cabozantinib could affect bone microenvironment in normal condition highlighting its potential role in mediating treatment responses, supporting the observations in patients with bone metastases in prostate cancer. In addition, other factors of the tumor microenvironment (including myeloid cells or microphages) are likely critical in promoting metastasis and a potential effect of cabozantinib on the tumor microenvironment needs further investigations $(26,41)$.

Our results with pan-VEGFR inhibition in vitro and in vivo support previously raised concerns that a more aggressive pattern of tumor cells may occur with anti-angiogenic therapies (42-44) through adaptive mechanisms such as activation of alternative pro-angiogenic signaling pathways such as $\mathrm{HGF} / \mathrm{c}-\mathrm{MET}$ and SRC signaling that may result in enhanced invasiveness and metastases. Dual VEGFR2 and MET inhibiting antitumor activity was mediated by anti-angiogenic, anti-migratory and pro-apoptotic effects, and associated with reduction of metastatic spread supporting the hypothesis that inhibition of a larger spectrum of targets beyond the VEGF pathway may help to circumvent the resistance to more selective angiogenesis blockade. The enhanced aggressiveness associated with HGF signaling may be considered in the further development of anti-angiogenic treatments in neuroblastoma.

\section{Acknowledgements}

We are grateful to the Platform of Preclinical Evaluation (PFEP) and Dr Patrick Gonin for providing immunocompromized mice and health care of animals as well as Dr Valérie Rouffiac of the Platform of Cell Imaging (PFIC) for their advice in bioluminescence imaging and Dr Ingrid Leguerney for ultrasound imaging. We thank Carole Lecinse for critical reading of the manuscript. The present study was supported by a grant from 'Fédération Enfants et Santé' and the 'Société Française des Cancers de l'Enfant.

\section{References}

1. Birchmeier C and Gherardi E: Developmental roles of HGF/SF and its receptor, the c-Met tyrosine kinase. Trends Cell Biol 8: 404-410, 1998

2. Grant DS, Kleinman HK, Goldberg ID, Bhargava MM, Nickoloff BJ, Kinsella JL, Polverini P and Rosen EM: Scatter factor induces blood vessel formation in vivo. Proc Natl Acad Sci USA 90: 1937-1941, 1993.

3. Zhang L, Yang N, Park JW, Katsaros D, Fracchioli S, Cao G, O'Brien-Jenkins A, Randall TC, Rubin SC and Coukos G: Tumor-derived vascular endothelial growth factor up-regulates angiopoietin-2 in host endothelium and destabilizes host vasculature, supporting angiogenesis in ovarian cancer. Cancer Res 63: 3403-3412, 2003.
4. Sulpice E, Ding S, Muscatelli-Groux B, Bergé M, Han ZC, Plouet J, Tobelem G and Merkulova-Rainon T: Cross-talk between the VEGF-A and HGF signalling pathways in endothelial cells. Biol Cell 101: 525-539, 2009.

5. Dong G, Chen Z, Li ZY, Yeh NT, Bancroft CC and Van Waes C: Hepatocyte growth factor/scatter factor-induced activation of MEK and PI3K signal pathways contributes to expression of proangiogenic cytokines interleukin- 8 and vascular endothelial growth factor in head and neck squamous cell carcinoma. Cancer Res 61: 5911-5918, 2001.

6. Dong G, Lee TL, Yeh NT, Geoghegan J, Van Waes C and Chen Z: Metastatic squamous cell carcinoma cells that overexpress c-Met exhibit enhanced angiogenesis factor expression, scattering and metastasis in response to hepatocyte growth factor. Oncogene 23: 6199-6208, 2004.

7. Abounader R, Lal B, Luddy C, Koe G, Davidson B, Rosen EM and Laterra J: In vivo targeting of SF/HGF and c-met expression via U1snRNA/ribozymes inhibits glioma growth and angiogenesis and promotes apoptosis. FASEB J 16: 108-110, 2002.

8. Abounader R and Laterra J: Scatter factor/hepatocyte growth factor in brain tumor growth and angiogenesis. Neuro Oncol 7: 436-451, 2005.

9. Goyal L, Muzumdar MD and Zhu AX: Targeting the HGF/c-MET pathway in hepatocellular carcinoma. Clin Cancer Res 19: 2310-2318, 2013.

10. Kawakami H, Okamoto I, Okamoto W, Tanizaki J, Nakagawa K and Nishio K: Targeting MET amplification as a new oncogenic driver. Cancers (Basel) 6: 1540-1552, 2014.

11. Sköldenberg EG, Larsson A, Jakobson A, Hedborg F, Kogner P, Christofferson $\mathrm{RH}$ and Azarbayjani F: The angiogenic growth factors HGF and VEGF in serum and plasma from neuroblastoma patients. Anticancer Res 29: 3311-3319, 2009.

12. Hecht M, Schulte JH, Eggert A, Wilting J and Schweigerer L: The neurotrophin receptor TrkB cooperates with c-Met in enhancing neuroblastoma invasiveness. Carcinogenesis 26: 2105-2115, 2005.

13. Hecht M, Papoutsi M, Tran HD, Wilting J and Schweigerer L: Hepatocyte growth factor/c-Met signaling promotes the progression of experimental human neuroblastomas. Cancer Res 64 : 6109-6118, 2004.

14. Pennacchietti S, Michieli P, Galluzzo M, Mazzone M, Giordano S and Comoglio PM: Hypoxia promotes invasive growth by transcriptional activation of the met protooncogene. Cancer Cell 3: 347-361, 2003.

15. Sennino $B$, Ishiguro-Oonuma $T$, Wei $Y$, Naylor $R M$, Williamson CW, Bhagwandin V, Tabruyn SP, You WK, Chapman HA, Christensen JG, et al: Suppression of tumor invasion and metastasis by concurrent inhibition of c-Met and VEGF signaling in pancreatic neuroendocrine tumors. Cancer Discov 2: 270-287, 2012.

16. Qian F, Engst S, Yamaguchi K, Yu P, Won KA, Mock L, Lou T, Tan J, Li C, Tam D, et al: Inhibition of tumor cell growth, invasion, and metastasis by EXEL-2880 (XL880, GSK1363089), a novel inhibitor of HGF and VEGF receptor tyrosine kinases. Cancer Res 69: 8009-8016, 2009.

17. Huynh H, Ong R and Soo KC: Foretinib demonstrates anti-tumor activity and improves overall survival in preclinical models of hepatocellular carcinoma. Angiogenesis 15: 59-70, 2012.

18. Yakes FM, Chen J, Tan J, Yamaguchi K, Shi Y, Yu P, Qian F, Chu F, Bentzien F, Cancilla B, et al: Cabozantinib (XL184), a novel MET and VEGFR2 inhibitor, simultaneously suppresses metastasis, angiogenesis, and tumor growth. Mol Cancer Ther 10: 2298-2308, 2011.

19. Zhang Y, Guessous F, Kofman A, Schiff D and Abounader R: XL-184, a MET, VEGFR-2 and RET kinase inhibitor for the treatment of thyroid cancer, glioblastoma multiforme and NSCLC. IDrugs 13: 112-121, 2010.

20. Torres KE, Zhu QS, Bill K, Lopez G, Ghadimi MP, Xie X, Young ED, Liu J, Nguyen T, Bolshakov S, et al: Activated MET is a molecular prognosticator and potential therapeutic target for malignant peripheral nerve sheath tumors. Clin Cancer Res 17: 3943-3955, 2011.

21. Navis AC, Bourgonje A, Wesseling P, Wright A, Hendriks W, Verrijp K, van der Laak JA, Heerschap A and Leenders WP: Effects of dual targeting of tumor cells and stroma in human glioblastoma xenografts with a tyrosine kinase inhibitor against c-MET and VEGFR2. PLoS One 8: e58262, 2013.

22. Bentzien F, Zuzow M, Heald N, Gibson A, Shi Y, Goon L, Yu P, Engst S, Zhang W, Huang D, Zhao L, et al: In vitro and in vivo activity of cabozantinib (XL184), an inhibitor of RET, MET, and VEGFR2, in a model of medullary thyroid cancer. Thyroid 23: $1569-1577,2013$. 
23. You WK, Sennino B, Williamson CW, Falcón B, Hashizume H Yao LC, Aftab DT and McDonald DM: VEGF and c-Met blockade amplify angiogenesis inhibition in pancreatic islet cancer. Cancer Res 71: 4758-4768, 2011.

24. Xiang Q, Chen W, Ren M, Wang J, Zhang H, Deng DY, Zhang L, Shang C and Chen Y: Cabozantinib suppresses tumor growth and metastasis in hepatocellular carcinoma by a dual blockade of VEGFR2 and MET. Clin Cancer Res 20: 2959-2970, 2014.

25. Kurzrock R, Sherman SI, Ball DW, Forastiere AA, Cohen RB, Mehra R, Pfister DG, Cohen EE, Janisch L, Nauling F, et al: Activity of XL184 (Cabozantinib), an oral tyrosine kinase inhibitor, in patients with medullary thyroid cancer. J Clin Oncol 29: 2660-2666, 2011.

26. Smith DC, Smith MR, Sweeney C, Elfiky AA, Logothetis C, Corn PG, Vogelzang NJ, Small EJ, Harzstark AL, Gordon MS, et al: Cabozantinib in patients with advanced prostate cancer: Results of a phase II randomized discontinuation trial. J Clin Oncol 31: 412-419, 2013.

27. Zhu K, Kong X, Zhao D, Liang Z and Luo C: c-MET kinase inhibitors: A patent review (2011-2013). Expert Opin Ther Pat 24: 217-230, 2014

28. Saylor PJ, Lee RJ and Smith MR: Emerging therapies to prevent skeletal morbidity in men with prostate cancer. J Clin Oncol 29: 3705-3714, 2011

29. Agarwal N, Sonpavde G and Sternberg CN: Novel molecular targets for the therapy of castration-resistant prostate cancer. Eur Urol 61: 950-960, 2011

30. Drilon A, Wang L, Hasanovic A, Suehara Y, Lipson D, Stephens P, Ross J, Miller V, Ginsberg M, Zakowski MF, et al: Response to Cabozantinib in patients with RET fusion-positive lung adenocarcinomas. Cancer Discov 3: 630-635, 2013.

31. Choueiri TK, Pal SK, McDermott DF, Morrissey S, Ferguson KC, Holland J, Kaelin WG and Dutcher JP: A phase I study of cabozantinib (XL184) in patients with renal cell cancer. Ann Oncol 25: 1603-1608, 2014

32. Elisei R, Schlumberger MJ, Müller SP, Schöffski P, Brose MS Shah MH, Licitra L, Jarzab B, Medvedev V, Kreissl MC, et al: Cabozantinib in progressive medullary thyroid cancer. J Clin Oncol 31: 3639-3646, 2013.

33. Daudigeos-Dubus E, LE Dret L, Rouffiac V, Bawa O, Leguerney I, Opolon P, Vassal G and Geoerger B: Establishment and characterization of new orthotopic and metastatic neuroblastoma models. In Vivo 28: 425-434, 2014.
34. Daudigeos-Dubus E, Le Dret L, Lanvers-Kaminsky C, Bawa O, Opolon P, Vievard A, Villa I, Pagès M, Bosq J, Vassal G, et al: Regorafenib: Antitumor activity upon mono and combination therapy in preclinical pediatric malignancy models. PLoS One 10: e0142612, 2015

35. Rössler J, Monnet Y, Farace F, Opolon P, Daudigeos-Dubus E, Bourredjem A, Vassal G and Geoerger B: The selective VEGFR1-3 inhibitor axitinib (AG-013736) shows antitumor activity in human neuroblastoma xenografts. Int J Cancer 128: 2748-2758, 2011

36. Sierra JR and Tsao MS: c-MET as a potential therapeutic target and biomarker in cancer. Ther Adv Med Oncol 3 (Suppl): S21-S35, 2011.

37. Zhang L, Scorsone K, Woodfield SE and Zage PE: Sensitivity of neuroblastoma to the novel kinase inhibitor cabozantinib is mediated by ERK inhibition. Cancer Chemother Pharmacol 76: 977-987, 2015.

38. Shojaei F, Simmons BH, Lee JH, Lappin PB and Christensen JG: $\mathrm{HGF} / \mathrm{c}-$ Met pathway is one of the mediators of sunitinib-induced tumor cell type-dependent metastasis. Cancer Lett 320: 48-55, 2012.

39. Huveldt D, Lewis-Tuffin LJ, Carlson BL, Schroeder MA, Rodriguez F, Giannini C, Galanis E, Sarkaria JN and Anastasiadis PZ: Targeting Src family kinases inhibits bevacizumab-induced glioma cell invasion. PLoS One 8: e56505, 2013.

40. Sengupta S, Gherardi E, Sellers LA, Wood JM, Sasisekharan R and Fan TP: Hepatocyte growth factor/scatter factor can induce angiogenesis independently of vascular endothelial growth factor. Arterioscler Thromb Vasc Biol 23: 69-75, 2003.

41. Haider MT, Hunter KD, Robinson SP, Graham TJ, Corey E, Dear TN, Hughes R, Brown NJ and Holen I: Rapid modification of the bone microenvironment following short-term treatment with Cabozantinib in vivo. Bone 81: 581-592, 2015.

42. Bergers $G$ and Hanahan D: Modes of resistance to anti-angiogenic therapy. Nat Rev Cancer 8: 592-603, 2008.

43. Bottsford-Miller JN, Coleman RL and Sood AK: Resistance and escape from antiangiogenesis therapy: Clinical implications and future strategies. J Clin Oncol 30: 4026-4034, 2012.

44. Giuliano S and Pagès G: Mechanisms of resistance to antiangiogenesis therapies. Biochimie 95: 1110-1119, 2013. 